Precious L. Barnes*, DO, MS, MS, Frank J. Casella, BS, Hilda Lai, MS, Olavi Airaksinen, MD, PhD and Michael L. Kuchera, DO, FAAO, FNAOME

\title{
Retention of tissue texture change after cervical muscle energy and high velocity low amplitude intervention: implications for treatment intervals
}

https://doi.org/10.1515/jom-2021-0187

Received July 18, 2021; accepted October 13, 2021;

published online January 24, 2022

\begin{abstract}
Context: When choosing to incorporate osteopathic manipulative treatment (OMT) into a patient's care, the risk-tobenefit ratio, the choice of treatment technique, as well as the frequency of treatments are always taken into consideration. This has been even more important during the COVID-19 pandemic, in which social distancing has been the best preventative measure to decrease exposure. By increasing treatment intervals, one could not only limit possible exposure/spread of viruses but also decrease the overall cost to the system as well as to the individual. This is an expansion of a previous study in which quantifiable changes in cervical hysteresis characteristics post-OMT were documented utilizing a durometer (Ultralign SA201 ${ }^{\circledR}$; Sigma Instruments; Cranberry, PA USA). This study compared two treatment modalities, muscle energy (ME) and high-velocity lowamplitude (HVLA) postcervical treatment. Subjects in this study were allowed to re-enroll, provided that they could be treated utilizing the alternate treatment modality. By allowing repeat subjects, analysis of the data for lasting effects of OMT could be observed.
\end{abstract}

\footnotetext{
*Corresponding author: Precious L. Barnes, DO, MS, MS, Eastern University of Finland, Kuopio, Finland; and Family Medicine/ Neuromusculoskeletal Medicine Hospitalist, Skagit Regional Hospitals, 1918 98th Ave SE, Lake Stevens, 98258 Mount Vernon, WA USA, E-mail: preciousba@pcom.edu

Frank J. Casella, BS and Hilda Lai, MS, Philadelphia College of Osteopathic Medicine, Philadelphia, PA, USA

Olavi Airaksinen, MD, PhD, Eastern University of Finland, Kuopio, Finland

Michael L. Kuchera, DO, FAAO, FNAOME, Marian University College of Osteopathic Medicine, Indianapolis, IN, USA
}

Objectives: To determine whether a significant change in cervical hysteresis would be observed after each treatment regardless of a short treatment interval.

Methods: A total of 34 subjects were retrospectively noted to be repeat subjects from a larger, 213-subject study. These 34 subjects were repeat participants who were treated with two different direct-treatment modalities 7-10 days apart. Each subject was randomly assigned to receive a singlesegmental ME or HVLA treatment technique directed toward a cervical (C) segment (C3-C5 only). Subjects were objectively measured pretreatment in all cervical segments utilizing the Ultralign SA20 ${ }^{\circledR}$, then treated with cervical OMT to a single segment, and finally reassessed at all cervical levels with the Ultralign SA201 ${ }^{\circledR}$ posttreatment to assess for change in cervical hysteresis.

Results: Statistically significant or suggestive changes (p-values $0.01-0.08$ ) with good clinical effect size (0.30 or greater) were noted in all four components of the Ultralign SA201 ${ }^{\circledR}$ at multiple cervical levels after the first treatment, but only one component (frequency) had a statistically significant change after the second treatment (AA cervical level, p-value 0.01) with good clinical effect size (0.45). However, when comparing the post-first-treatment values to the pre-second-treatment values, no statistically significant differences (p-value 0.10 or higher) were observed between them.

Conclusions: Statistically significant changes were noted after the first treatment; however, when comparing cervical hysteresis changes after the first treatment to the cervical hysteresis values prior to the second treatment delivered 7-10 days later, there were no statistically significant or suggestive changes. This data suggest that several postOMT changes noted after the first treatment were still in effect and may indicate that follow-up visits for direct manipulation may be deferred for a least two weeks.

Keywords: COVID-19; hysteresis; hysteresis changes; neuromusculoskeletal medicine; NMM; OMM; OMT; osteopathic manipulative medicine; treatment intervals. 
The age-old question of when a patient should return for a follow-up osteopathic manipulative treatment (OMT) is frequently debated. In the current COVID-19 pandemic climate, the answer to this question is even more relevant because social distancing [1] and masking are recommended to decrease the spread of the virus [2]. Even though more people are vaccinated and state-mandated masking is decreasing, the risk-to-benefit ratio continues to be evaluated when treating patients, and this includes the use of OMT. Empirically, many providers recommend that patients should follow up in 1-4 weeks, and some say that a patient could be treated daily. Most osteopathic physicians believe that the body needs time to readjust after a treatment [3] and that the effects of the treatment can last $[4,5]$ for several days in between treatment sessions or that the body takes over and can continue healing itself [6]. This can be challenging to measure because interpreting the true nature of a treatment is sometimes difficult with subjective data $[7,8]$. However, with the assistance of the Ultralign SA201 ${ }^{\circledR}$ (Sigma Instruments; Cranberry, PA), we attempted to shed some light on this question by documenting quantifiable hysteresis changes post-OMT [9].

In a previous foundational study [10], quantifiable changes in cervical hysteresis characteristics post-OMT were documented utilizing a durometer (The Ultralign SA201 $\left.{ }^{\circledR}\right)$ [11]. In that study $(\mathrm{n}=240)$, the Ultralign SA201 ${ }^{\circledR}$ was able to document objective cervical hysteresis changes from multiple OMT modalities when compared to a sham group [10]. Subsequently, a follow-up preliminary study $(\mathrm{n}=213)$ was designed to narrow its focus by comparing two direct treatment modalities, Muscle Energy (ME) and high-velocity low-amplitude (HVLA) directed to a single segment of the cervical (C) spine. This study allowed subjects to re-enroll, provided that they could be treated utilizing the alternate treatment modality. By allowing repeat subjects, further analysis of the data could be performed to assess for lasting effects from the previous treatment as well as to further changes made after the second treatment within a short period of time. Of note, it was observed in the foundational study $(n=240)$ that the acts of hysteresis measurements and palpation were not noted to make a difference in the objective readings of the Ultralign SA201 ${ }^{\circledR}$; therefore, a sham arm was not utilized for the follow-up parent study $(\mathrm{n}=213)$.

The Ultralign SA20 ${ }^{\circledR}$ system is a durometer capable of analyzing and quantifying hysteresis $[12,13]$ changes in tissue texture at various spinal levels [14]. The system does this by measuring deformation and recoil of tissue responses (hysteresis) [12] to a specific piezoelectric impulse and measures those changes in durometers [14]. There are four components utilized to calculate a durometer: motoricity, mobility, frequency, and fixation. Together, these components produce a parabolic curve, and each portion of the curve is considered to measure a portion of somatic dysfunction [10]. Motoricity represents the overall dysfunction of a segment. Mobility corresponds to the range of motion for a segment. Frequency is the time it takes to meet either a restrictive or physiologic barrier. Finally, fixation indicates the resistance within the tissues $[10,11]$. These four components [10] are what the Ultralign SA201 ${ }^{\circledR}[11]$ utilizes in the analysis [14] and documentation of changes in cervical hysteresis after OMT [15].

Our hypothesis for this study is that an objective change would be observed in repeat subjects after each direct treatment technique regardless of the short interval between treatment.

\section{Methods}

In this Philadelphia College of Osteopathic Medicine IRB-approved study (H11-013), 34 subjects were extracted from a larger, 213-subject study. All subjects were allowed to reenroll in the study as long as they had no contraindications to having the alternative treatment method performed. The study was performed on a medical school campus over a six-month period and recruitment took place via posted flyers around campus. Subjects who were students were offered one point of extra credit for participating in at least two campus studies; no other form of compensation was offered to nonstudents or students who only participated in one campuswide study. There were only 34 subjects with repeat hysteresis measurements for comparison. Each of the 34 subjects was a relatively healthy medical student with neck pain ranging from 1 to 10 on a 10-point analog pain scale, with one being the least painful and 10 being the highest reported pain level. Subjects were randomly assigned to receive single-segmental ME or HVLA by blindly choosing the direct method technique type out of a bag. An even number of options were placed in the bag, and prior to treatment, the subjects would choose one of the options. The technique options were labeled \#1 (for HVLA) and \#2 (for ME). The subjects were not informed as to which treatment technique that number corresponded. Treatment was only directed toward cervical levels C3, C4, or C5. According to the foundational study [10] $(n=240)$, in this population, the cervical levels $\mathrm{C} 3-\mathrm{C} 5$ were found to most commonly have significant somatic dysfunction [16]; therefore, treatment was limited to these three segments. The cervical site treated was considered by the treating physician (MLK) to have the most significant somatic dysfunction based on standard osteopathic palpatory diagnosis and which prioritized restricted motion and tissue texture abnormalities $[6,16]$. Each repeat subject was reexamined and treated with the other direct treatment method 7-10 days after the first treatment. All subjects were objectively measured utilizing the Ultralign SA201 ${ }^{\circledR}$ (PLB) before and after each manual technique. To ensure consistency with the measurements taken by the Ultralign SA201 ${ }^{\circledR}$, each cervical area 


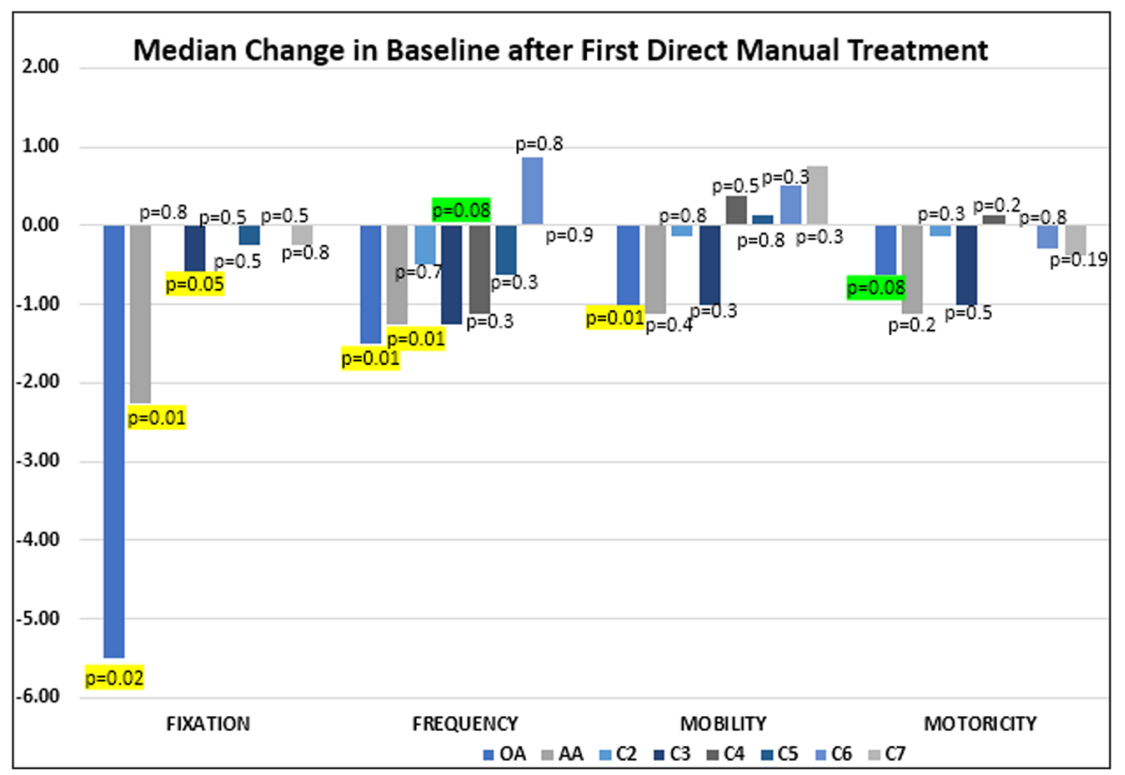

Figure 1: Changes in median values compared to baseline durometer measurements after the first direct manual treatment. The statistically significant values $(p \leq 0.05)$ are highlighted in yellow, and the statistically suggestive values $(p \leq 0.1)$ are highlighted in green.
(OA-C7) was measured four times pre- and posttreatment. The average of the four measurements for each durometer component was utilized in the final statistical analysis.

To be included in this study, the subject had to be a relatively healthy individual between the ages of 18 and 65 years who were assessed by palpatory diagnosis to have a manipulable somatic dysfunction, and willing to accept either ME or HVLA treatment to the cervical spine. Subjects were excluded from the study if they were outside of the age range, had a history of abnormal findings on a brain or cervical CT/MRI scan, significant scoliosis, or had a history of neoplastic disease, connective tissue disorder, or systemic disease such as rheumatoid arthritis, lupus, or ankylosing spondylitis. Subjects were also excluded if they had a significant psychiatric history or a history of symptomatic osteoarthritis of the cervical spine as documented in previous imaging studies or as evidenced by palpation and provocative test during the structural exam prior to treatment. A printed copy of the informed consent was signed and dated by each participant and collected by the research staff before any diagnostic or intervention procedure was performed. Subjects were given the opportunity to ask any questions prior to any procedures to ensure that they did not meet any of the exclusion criteria as well. This was a partially blinded study because the Ultralign SA201 ${ }^{\circledR}$ physician (PLB) and the osteopathic treating physician (MLK) remained blinded to the other's findings throughout the study.

Univariate analysis testing was utilized as this statistical assessment because it takes data, summarizes it, and finds patterns within the data [17]. Univariate analysis testing also documents whether there is a statistically suggestive $(\mathrm{p} \leq 0.1)$ or a statistically significant $(\mathrm{p} \leq 0.05)$ treatment or a component measured. Clinical effect size analysis was also chosen because it measures the strength of the relationship between two variables in a statistical population [18] along with the effectiveness of use in the clinic. Any value of moderate clinical effect size or greater is considered a good indicator of clinical change. A moderate clinical effect size is indicated by any value greater than or equal to 0.30 , and a strong clinical effect size is considered as any value greater than or equal to 0.70 . These two measurements illustrate the usefulness in the clinic as well as the validity of the components being measured. Finally, one-factor analysis testing was utilized to look for statistically significant differences between the first and second treatments [19]. All statistical analysis was performed by an accredited PhD-level statistician.

\section{Results}

Out of the 213 subjects, only 34 had repeat data for comparison ( 23 females and 11 males). The age range for these 34 subjects was 23-27 years (median age, 24 years; mean age, 24.2 years). The repeat subjects $(n=34)$ were evaluated by palpation and the SA201 ${ }^{\circledR}$ at cervical levels OA-C7. The median hysteresis value in each durometer component was compared to the baseline pretreatment values to assess for any tissue texture changes. Utilizing the univariate analysis test and clinical effect size, statistically significant or statistically suggestive hysteresis changes from baseline were noted post-OMT in at least one cervical level in all four components of the Ultralign SA201 ${ }^{\circledR}$. Fixation (OA $[\mathrm{p}=0.02$; confidence interval $(\mathrm{CI})-7.70$ to -0.45 ; clinical effect 0.39], AA [p=0.01; CI -2.92 to -0.37 ; clinical effect 0.45 ], C3 [p-value $=0.05$; CI -2.25 to 0.03; clinical effect $0.34]$ ), Frequency (OA [p-value $=0.01 ; \mathrm{CI}-3.19$ to -0.35 ; clinical effect 0.44], AA [p-value=0.01; CI -1.99 to -0.29 ; clinical effect 0.47], C3 [p=0.08; CI -2.29 to 0.14; clinical effect 0.31]), Mobility (OA [p=0.01; CI -2.51 to -0.29 ; clinical effect 0.44$]$ ), and Motoricity (OA [p-value=0.08; CI -2.44 to 0.16; clinical effect 0.31]) (Figures 1 and 2).

However, following the second treatment, only one component of the Ultralign SA201 ${ }^{\circledR}$ showed a statistically significant and statistically suggestive change from baseline: Frequency (AA $[\mathrm{p}=0.014$; CI -1.61 to -0.20 ; clinical 

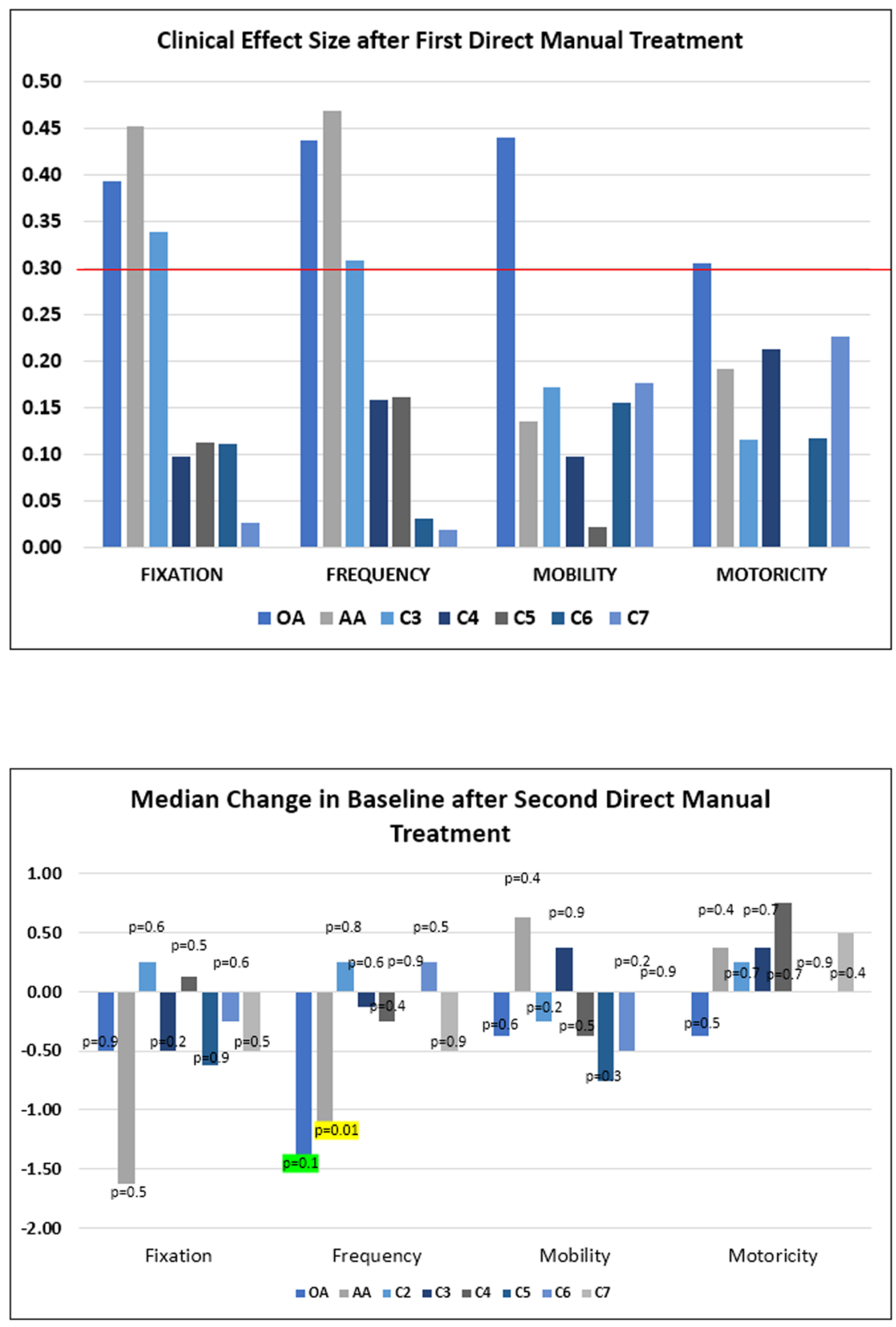

Figure 2: Red line depicting good clinical effect size $(\geq 0.30)$ from the first treatment with a direct OMT technique. Any number at or above the red line demonstrates good clinical effect.
Figure 3: Changes in median values compared to baseline measurements after the second direct manual treatment delivered 7-10 days after the first intervention. Statistically significant values $(p \leq 0.05)$ highlighted in yellow and statistically suggestive values $(p \leq 0.1)$ highlighted in green. effect 0.45 ], OA [p=0.12; CI -2.15 to 0.29 ; minimal clinical effect 0.27]) (Figures 3 and 4).

When observing if there was a change from baseline between the first posttreatment values compared to the values prior to the second treatment utilizing a one-factor analysis of variance test, however, no statistically significant difference in objective durometer-measured tissue texture change was noted between the end of the first treatment and the beginning of the second treatment (Figure 5).

\section{Discussion}

When evaluating the repeat subjects only, changes were observed post-OMT after the first treatment with good clinical effect size in at least one cervical level in all four durometer components, yet only minimal changes were noted after the second treatment. However, when comparing the first posttreatment values to the values taken 7-10 days before the second treatment, there is no statistically significant difference between the two values. This finding suggests that 

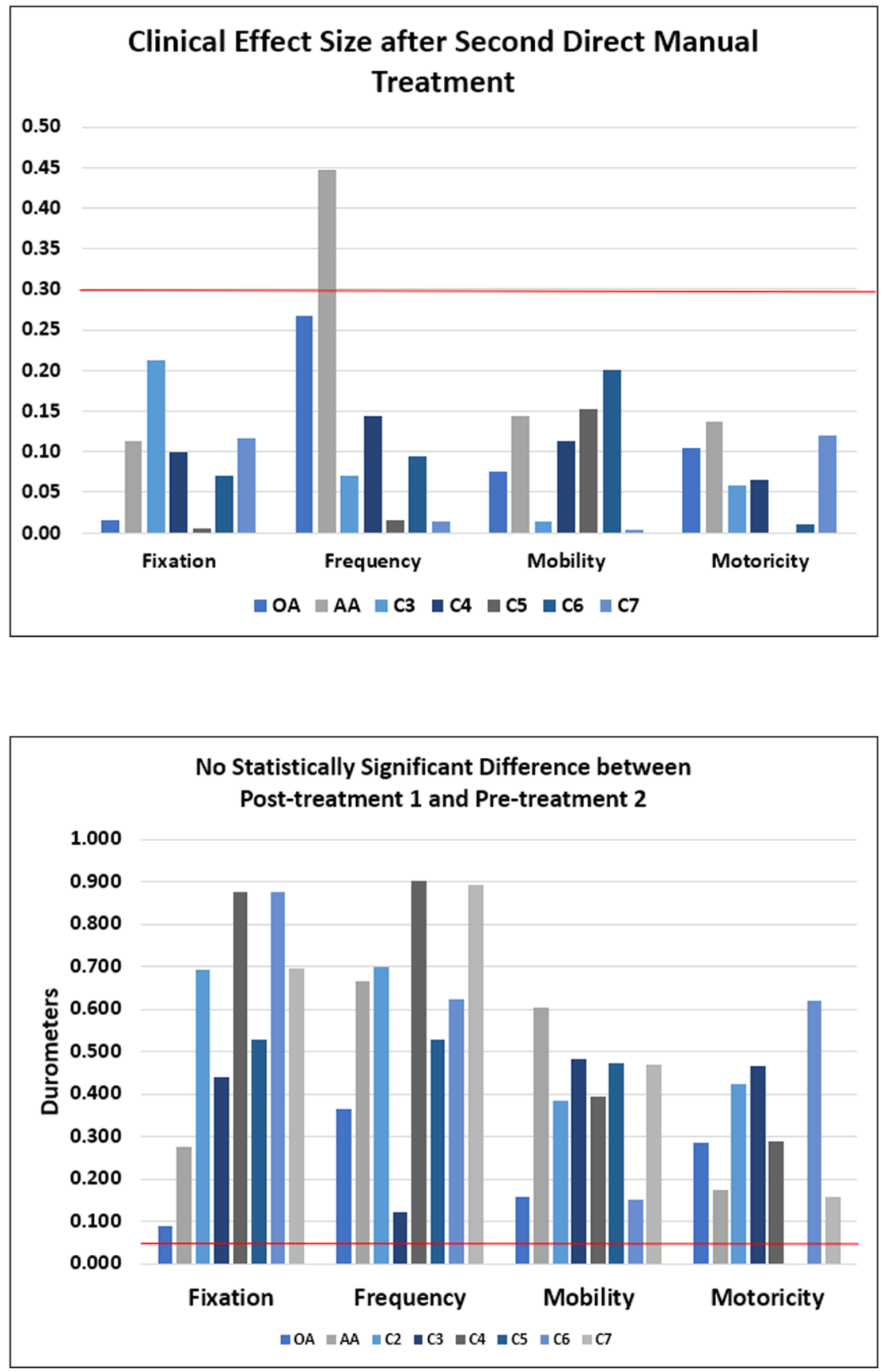

Figure 4: Red line depicting good clinical effect size $(\geq 0.30)$ after a second direct OMT technique delivered 7-10 days after the first intervention. Any number at or above the red line demonstrates good clinical effect. Only one cervical level in one durometer component demonstrated good clinical effect.
Figure 5: Red line depicting p-value of 0.05 . Any number at or below this line demonstrates statistical significance. There are no statistically significant changes between post-treatment 1 and pretreatment 2 (7-10 days later), suggesting that the changes made after treatment 1 still seem to be in effect before the administration of treatment 2 . hysteresis changes documented after the first direct treatment were retained for at least 7-10 days and did not improve further in response to a second direct treatment applied within this time frame. As the first treatment was still in effect, it would seem that subsequent patient follow-ups for direct OMT of less than two weeks may not be an optimal treatment interval.

Although only somatic dysfunction of C3, C4, or C5 was treated, it should be noted that hysteresis change was most noted in the superior cervical segments (OA-C3) and that no changes were noted in the lower cervical segments (C6-C7). The superior cervical segment could represent a tensegrity effect in this relatively healthy population.

In the context of this paper, one limitation is that this study only looked at two direct OMT techniques. Sicker patients and those unable to tolerate direct methods may benefit from different techniques delivered more or less frequently. Further studies should be conducted to assess this possibility. 
Another limitation in generalizing conclusions from this study is that osteopathic practitioners do not typically apply a single technique with a clinical goal of simply addressing tissue texture abnormalities in a single spinal region. Further studies are needed to integrate objective durometer findings related to other regions and other clinical goals.

Some other limitations to the study arise from its small subgroup sample. We were also limited in the fact that the Ultralign SA201 ${ }^{\circledR}$ was only able to measure hysteresis change, which is only one aspect of somatic dysfunction. Although the researchers were hoping to generalize this study, an uneven number of males and females were enrolled in this subgroup.

In any treatment intervention, clinical decisions include both cost and risk-to-benefit considerations. Factors affecting these considerations include the duration of the treatment's effect and the frequency of its application. HVLA and ME are quick and succinct techniques that decrease the time it takes to treat a patient and thus the physician-patient contact-time. As a potential added bonus, increased treatment intervals could decrease the risk of exposure to COVID-19 because the CDC recommends a quarantine period of 10-14 days to prevent transmission of the virus [2].

\section{Conclusions}

Treatment of palpated somatic dysfunction in the midcervical spine (C3-C5) on a first visit utilizing directmethod OMT technique resulted in significant and suggestive objective durometer changes that were retained when remeasured 7-10 days later. Residual somatic dysfunction palpated in that region 7-10 days after the first intervention, and treated with a direct-method OMT technique, resulted in minimal further durometer change. By potentially limiting treatment intervals to two weeks or greater, patient and physician safety could be enhanced, and the cost of treatment could be more affordable to both the system and the individual.

Research funding: The Ultralign SA201 ${ }^{\circledR}$ machine was donated to the Human Performance and Biomechanics Laboratory at the Philadelphia College of Osteopathic Medicine by Sigma Instruments. No financial funding was given towards this research.

Author contributions: All authors provided substantial contributions to the conception and design, acquisition of data, or analysis and interpretation of the data; P.L.B.,
M.L.K., F.J.C., and H.L. provided a substantial contribution to the acquisition of data; P.L.B., M.L.K., O.A., and F.J.C. drafted the article or revised it critically for important intellectual content; all authors gave final approval of the version of the article to be published; and all authors agree to be accountable for all aspects of the work in ensuring that questions related to the accuracy or integrity of any part of the work are appropriately investigated and resolved.

Competing interests: None reported.

Ethical approval: This research was reviewed and approved by the Institutional Review Board (IRB) at the Philadelphia College of Osteopathic Medicine (IRB approval number: H11-013).

Informed consent: All participants in this study provided written informed consent prior to participation.

\section{References}

1. Centers for Disease Control and Prevention. Social distancing; 2021. https://www.cdc.gov/coronavirus/2019-ncov/preventgetting-sick/social-distancing.html [Accessed 14 Sep 2021].

2. Centers for Disease Control and Prevention. How to protect yourself \& others; 2021. https://www.cdc.gov/coronavirus/ 2019-ncov/prevent-getting-sick/prevention.html [Accessed 14 Sep 2021].

3. Hohenschurz-Schmidt DJ, Esteves JE, Thomson OP. Tensegrity and manual therapy practice: a qualitative study. Int J Osteopath Med 2016;21:5-18.

4. Kuchera ML, Kuchera WA. Osteopathic considerations in head, eye, ear, nose and throat (HEENT) disorders. Columbus, $\mathrm{OH}$ : Greyden Press; 2012.

5. Kuchera WA, Kuchera ML. Chapter 8: diagnosis and manipulative treatment: cervical region. In: Kuchera WA, Kuchera ML, editors. Osteopathic principles in practice, Revised 2nd ed. Columbus, $\mathrm{OH}$ : Original Works Greyden Press; 1994:571-90 pp.

6. Seffinger M. Foundations of osteopathic medicine, 4th ed. Philadelphia, PA: Wolters Kluwer; 2018.

7. Rogers FJ. A call for evidence-based medicine: evolving standards and practice. J Am Osteopath Assoc 2007;107:293-4.

8. Patijn J. Reproducibility protocol for diagnostic procedures in manual/musculoskeletal medicine. Manuel Med 2019;57:451-79.

9. Russell R. Diagnostic palpation of the spine: a review of procedures and assessment of their reliability. J Manipulative Physiol Therapeut 1983;6:181-3.

10. Barnes PL, Laboy F, Noto-Bell L, Ferencz V, Nelson J, Kuchera ML. A comparative study of cervical hysteresis characteristics after various osteopathic manipulative treatment (OMT) modalities. J Bodyw Mov Ther 2013;17:89-94.

11. SIGMA Instrument. What is the pro-adjuster/SIGMA instrument; 2021. https://www.adjustmentschiropractic.com/ultralign/ [Accessed 1 Mar 2021].

12. Sethna J. What's Hysteresis. Cornell Theory Center; 1999. http://www.lassp.cornell.edu/sethna/hysteresis/ WhatlsHysteresis.html [Accessed 9 Sep 2020]. 
13. Cohen AM, Stewart P, Warner MJ, Kuchera ML. Hysteresis as a measure of ankle dysfunction. J Am Osteopath Assoc 2005;105:22.

14. Zahornitzky W, Keusch R, Tilscher H, Beyer L. Treatment of upper cervical syndrome with functional computer-assisted reflex therapy (Spineliner). Manuel Med 2010;48:31-8.

15. DeBusk C. How neck pain significantly improved with spine aligner. Chiropractic Economics; 2017. https://www.chiroeco. com/spine-aligner-chiropractic-methods/ [Accessed 1 Mar 2021].

16. Giusti R. Glossary of osteopathic terminology, 3rd ed. Chevy Chase, MD: American Association of Colleges of Osteopathic Medicine; 2017.
17. Glen S. Univariate analysis: definition, examples. Statistics how to; 2021. https://www.statisticshowto.com/univariate/ [Accessed 1 Mar 2021].

18. Faraone SV. Understanding effect size: how it's measured and what it means. ADHD expert column series. Medscape Psychiatry; 2008. https://www.medscape.org/viewarticle/569729 [Accessed 1 Sep 2018].

19. National Institute of Standards and Technology a unit of the US Department of Commerce. 1.3.5.4. One-factor ANOVA. Engineering Statistics Handbook; 2013. https://www.itl.nist.gov/div898/ handbook/eda/section3/eda354.htm [Accessed 12 Feb 2021]. 